\begin{tabular}{cc}
\hline & COMPUTATIONAL RESEARCH PROGRESS IN APPLIED SCIENCE \& ENGINEERING (CRPASE) \\
& CRPASE: TRANSACTIONS OF MECHANICAL ENGINEERING \\
\hline
\end{tabular}

\title{
A Novel Reliability Calculation Method Based on Fuzzy Universal Generating Function
}

\author{
Huiying Gao ${ }^{1}$, Xiaoqiang Zhang ${ }^{1 *}$, Xiaoqiang Yang ${ }^{1}$, Bo Zheng ${ }^{1}$, Xin $\mathrm{Ma}^{2}$ \\ ${ }^{1}$ Aviation Engineering Institute, Civil Aviation Flight University of China, Guanghan, China \\ ${ }^{2}$ Air Traffic Management School, Civil Aviation Flight University of China, Guanghan, China
}

\begin{tabular}{ll}
\hline Keywords & Abstract \\
\cline { 2 - 3 } $\begin{array}{l}\text { Universal generating } \\
\text { function, }\end{array}$ & $\begin{array}{l}\text { The universal generating function (UGF) can be used for reliability calculation where the } \\
\text { Reliability, }\end{array}$ \\
$\begin{array}{l}\text { Fuzzy, } \\
\text { Multi-state system variables are discrete random variables. In some cases, the possible values of the } \\
\text { Uncertainties. }\end{array}$ & $\begin{array}{l}\text { variables are not crisp values, which may be fuzzy. This paper regards the possible values } \\
\text { of the system variables and the corresponding probability as triangular fuzzy numbers } \\
\text { (TFNs), a fuzzy reliability method based on fuzzy universal generating function (FUGF) is } \\
\text { proposed. The proposed method can deal with fuzzy reliability issues. A case study is } \\
\text { presented to illustrate the proposed method. Through the comparison between the required } \\
\text { reliability and the calculated value, whether the design scheme is feasible can be determined. }\end{array}$ \\
\hline
\end{tabular}

\section{Introduction}

The universal generating function (UGF) was firstly introduced by Ushakov [1] to reduce the computational complexity. Lisnianski [2] and Levitin [3-4] have done a lot of research work on it and the UGF method has been widely applied in reliability analysis and optimization of multi-state systems. The UGF technique can be used to determine the output performance distributions of multi-state systems (MSSs). By determining different composition operators, the performance distributions of MSSs with series, parallel, series-parallel and bridge structures were evaluated in [2-4]. Yeh $[5,6]$ developed UGF based methods to estimate the reliability of k-out-of-n acyclic multi-state node network (AMNN) and general multi-state node network, respectively. The UGF technique suggested in [7] was used to evaluate the service reliability of grid computing systems with star topology. The techniques for reliability evaluation of multistate weighted k-out-of-n systems were presented in [8] by using UGF approach and recursive algorithms. The UGF technique is also suitable for solving different MSS reliability optimization problems because the reliability index can be evaluated by a fast procedure. Levitin et al. [4] proposed a system redundancy optimization algorithm for MSS with series-parallel system structure by using UGF technique. In [9] the optimal allocation problem of multistate elements in networks was discussed. The algorithm for solving optimal service task partition and distribution in grid system with star topology was suggested in [10]. Golrokh et al. [10] proposed several optimization models for ecoefficiency evaluation. Kuo [12] summarized the state of the art of optimal reliability design, in which the UGF technique was acknowledged as the primary approach for solving MSS reliability assessment and optimization problems.

Although a lot of research work have been done on UGF, but they all treat the system variables as crisp values. Therefore, this paper proposes a reliability calculation method based on the FUGF, which can deal with issues contain fuzzy variables. The proposed method is an extension of the UGF technique and has a wider applicability.

\section{A Brief Review of Universal Generating Function}

\footnotetext{
* Corresponding Author: Xiaoqiang Zhang

E-mail address: xqzhanguestc@163.com
}

Received: 24 April 2021; Revised: 22 May 2021; Accepted: 25 May 2021 https://doi.org/10.52547/crpase.7.2.2335 
Suppose that a discrete random variable with finite possible values. The possible values and the corresponding probability can be denoted as

$$
\begin{aligned}
& \mathbf{x}=\left(x_{1}, x_{2}, \cdots, x_{n}\right) \\
& \mathbf{p}=\left(p_{1}, p_{2}, \cdots, p_{n}\right)
\end{aligned}
$$

where $p_{i}=\operatorname{Pr}\left(X=x_{i}\right), 1 \leq i \leq n$ and the mapping $x_{i} \rightarrow p_{i}$ is usually called the probability mass function (p.m.f). The p.m.f can be represented by a polynomial function of $z$ as

$u_{X}(z)=p_{1} z^{x_{1}}+p_{2} z^{x_{2}}+\cdots+p_{n} z^{x_{n}}=\sum_{i=1}^{n} p_{i} z^{x_{i}}$

Eq. (1) is so-called universal generating function of $X$. It can be easy found that the p.m.f and the UGF of a discrete random variable exists one-to-one correspondence. Considering $m$ independent discrete random variables $X_{1}, X_{2}, \cdots, X_{m}$ and the UGF of each variable is $u_{X_{1}}(z), u_{X_{2}}(z), \cdots, u_{X_{m}}(z)$, respectively. By defining the composition operator $\otimes$, the UGF of an arbitrary function $f\left(X_{1}, X_{2}, \cdots, X_{m}\right)$ can be expressed as

$$
u_{f}(z)=\otimes\left(u_{X_{1}}(z), u_{X_{2}}(z), \cdots, u_{X_{m}}(z)\right)
$$

According to Eq. (1), the UGF of each discrete random variable can be denoted as

$$
\begin{aligned}
& u_{X_{1}}(z)=\sum_{j_{1}=1}^{k_{1}} p_{1 j_{1}} z^{x_{1 j_{1}}} \\
& u_{X_{2}}(z)=\sum_{j_{2}=1}^{k_{2}} p_{2 j_{2}} z^{x_{2 j_{2}}} \\
& \vdots \\
& u_{X_{m}}(z)=\sum_{j_{m}=1}^{k_{m}} p_{m j_{m}} z^{x_{m j_{m}}}
\end{aligned}
$$

where $k_{1}, k_{2}, \cdots, k_{m}$ are the number of possible values of $X_{1}, X_{2}, \cdots, X_{m}$. Then Eq. (2) can be rewritten as

$$
\begin{aligned}
u_{f}(z) & =\otimes\left(u_{X_{1}}(z), u_{X_{2}}(z), \cdots, u_{X_{m}}(z)\right) \\
& =\otimes\left(\sum_{j_{1}=1}^{k_{1}} p_{1 j_{1}} z^{x_{1 j_{1}}}, \sum_{j_{2}=1}^{k_{2}} p_{2 j_{2}} z^{x_{2 j_{2}}}, \cdots, \sum_{j_{m}=1}^{k_{m}} p_{m j_{m}} z^{x_{m j_{m}}}\right) \\
& =\sum_{j_{1}=1}^{k_{1}} \sum_{j_{2}=1}^{k_{2}} \cdots \sum_{j_{m}=1}^{k_{m}}\left(\prod_{i=1}^{m} p_{i_{i}} z^{f\left(x_{1 j_{1}}, x_{2 j_{2}}, \cdots, x_{m j_{m}}\right)}\right)
\end{aligned}
$$

\section{The Proposed Reliability Calculation Method}

In some cases, the possible values and the corresponding probability are not crisp values, they may be measured as fuzzy values. Assume that the fuzzy variables $\tilde{S}_{1}, \tilde{S}_{2}, \cdots, \tilde{S}_{n}$, and they are independent from each other. The p.m.f are known as

$$
\begin{aligned}
& \tilde{\mathbf{s}}_{1}=\left(\tilde{s}_{11}, \tilde{s}_{12}, \cdots, \tilde{s}_{1 k_{1}}\right), \tilde{\mathbf{p}}_{1}=\left(\tilde{p}_{11}, \tilde{p}_{12}, \cdots, \tilde{p}_{1 k_{1}}\right) \\
& \tilde{\mathbf{s}}_{2}=\left(\tilde{s}_{21}, \tilde{s}_{22}, \cdots, \tilde{s}_{2 k_{2}}\right), \tilde{\mathbf{p}}_{2}=\left(\tilde{p}_{21}, \tilde{p}_{22}, \cdots, \tilde{p}_{2 k_{2}}\right) \\
& \vdots \\
& \tilde{\mathbf{s}}_{n}=\left(\tilde{s}_{n 1}, \tilde{s}_{n 2}, \cdots, \tilde{s}_{n k_{n}}\right), \tilde{\mathbf{p}}_{2}=\left(\tilde{p}_{n 1}, \tilde{p}_{n 2}, \cdots, \tilde{p}_{n k_{n}}\right)
\end{aligned}
$$

where $k_{1}, k_{2}, \cdots, k_{n}$ are the number of possible values of the fuzzy variables, respectively. Similarly, the fuzzy universal generating function (FUGF) of $\tilde{S}_{1}, \tilde{S}_{2}, \cdots, \tilde{S}_{n}$ can be obtained.

$$
\begin{aligned}
& u_{\tilde{S}_{1}}(z)=\sum_{i_{1}=1}^{k_{1}} \tilde{p}_{1 i_{1}} z^{\tilde{s}_{1 i_{1}}} \\
& u_{\tilde{S}_{2}}(z)=\sum_{i_{2}=1}^{k_{2}} \tilde{p}_{2 i_{2}} z^{\tilde{s}_{2 i_{2}}} \\
& \vdots \\
& u_{\tilde{S}_{n}}(z)=\sum_{i_{n}=1}^{k_{n}} \tilde{p}_{n i_{n}} z^{\tilde{s}_{n_{n}}}
\end{aligned}
$$

The FUGF of an arbitrary function $f\left(\tilde{S}_{1}, \tilde{S}_{2}, \cdots, \tilde{S}_{n}\right)$ with respect to $\tilde{S}_{1}, \tilde{S}_{2}, \cdots, \tilde{S}_{n}$ can be expressed as

$$
\begin{aligned}
\tilde{u}_{f}(z) & =\otimes\left(\tilde{S}_{1}, \tilde{S}_{2}, \cdots, \tilde{S}_{n}\right) \\
& =\otimes\left(\sum_{i_{1}=1}^{k_{1}} \tilde{p}_{1 i_{1}} z^{\tilde{s}_{i_{1}}}, \sum_{i_{2}=1}^{k_{2}} \tilde{p}_{2 i_{2}} z^{\tilde{s}_{2 i_{2}}}, \cdots, \sum_{i_{n}=1}^{k_{n}} \tilde{p}_{n i_{n}} z^{\tilde{s}_{n i_{n}}}\right) \\
& =\sum_{i_{1}=1}^{k_{1}} \sum_{i_{2}=1}^{k_{2}} \cdots \sum_{i_{n}=1}^{k_{n}}\left(\prod_{j=1}^{n} \tilde{p}_{j i_{j}} z^{f\left(\tilde{s}_{i_{1}}, \tilde{s}_{2 i_{2}}, \cdots, \tilde{s}_{n i_{n}}\right)}\right)
\end{aligned}
$$

Assume that the performance of a component or a system is $\tilde{g}=f\left(\tilde{S}_{1}, \tilde{S}_{2}, \cdots, \tilde{S}_{n}\right)$ and the desired performance (demand) is $\tilde{\omega}$. Defining a function $\tilde{r}=\tilde{g}-\tilde{\omega}$, then the component is reliable if $\tilde{r}>0$, whereas, failure occurs.

For a triangular fuzzy number (TFN) $\tilde{x}$ represented as $(m, l, n)$, the membership function is

$$
\mu_{\tilde{x}}(x)= \begin{cases}0, & x<m \\ \frac{x-m}{l-m}, & m \leq x \leq l \\ \frac{n-x}{n-l}, & l<x \leq n \\ 0, & x>n\end{cases}
$$

The probability of $\tilde{x}>0$ is defined as

Case 1: $m>0$, it means that the minimum possible value $x_{\min }$ satisfies $x_{\min }>0$, and the probability of $\tilde{x}>0$ is denoted as $r_{r e l}=1$;

Case 2: $n<0$, it means that the maximum possible value $x_{\max }$ satisfies $x_{\max }<0$, and the probability of $\tilde{x}>0$ is denoted as $r_{\text {rel }}=0$

Case 3: $m<0, n>0$, it means that the possible value can be a positive number or a negative number, and the probability of $\tilde{x}>0$ is denoted as $r_{r e l}=\int_{0}^{n} \mu_{\tilde{x}}(x) \mathrm{d} x / \int_{m}^{n} \mu_{\tilde{x}}(x) \mathrm{d} x$.

The fuzzy reliability of the component can be calculated as 


$$
\begin{aligned}
\tilde{R} & =\otimes^{\prime}\left(\tilde{u}_{f}(z), \tilde{\omega}\right) \\
& =\otimes^{\prime}\left(\sum_{i_{1}=1}^{k_{1}} \sum_{i_{2}=1}^{k_{2}} \cdots \sum_{i_{n}=1}^{k_{n}}\left(\prod_{j=1}^{n} \tilde{p}_{j i_{j}} z^{f\left(\tilde{s}_{i_{i}}, \tilde{s}_{2 i_{2}}, \cdots, \tilde{s}_{n_{n_{n}}}\right)}\right), \tilde{\omega}\right) \\
& =\otimes^{\prime}\left(\sum_{i=1}^{M} \tilde{p}_{i} z^{\tilde{s}_{i}}, \tilde{\omega}\right) \\
& =\left\{R, \mu_{\tilde{R}}(R) \mid R=\sum_{i=1}^{M} p_{i} r_{r e l}^{i}, p_{i} \in P_{i}\right\}
\end{aligned}
$$

\section{Case Study}

Consider a flow transmission system as Figure 1 which consists of three elements (pipes). The flow is transmitted from left to right and the performance of the pipe is measured by their transmission capacity (tons of per minute). It is supposed that elements 1 and 2 have three states: a state of total failure corresponding to a capacity of 0 , a state of full capacity represented by a crisp value, and a state of partial failure represented by a TFN. The element 3 only has two states: one is a state of total failure and the other is a state of full capacity represented by a crisp value. It is also assumed that the state probabilities of each element are represented as TFNs. The parameters for the elements are shown in Table 1. Suppose that the system demand ( $\tilde{\omega})$ is estimated as a TFN, which is represented as $(1.3,1.4,1.5)$.

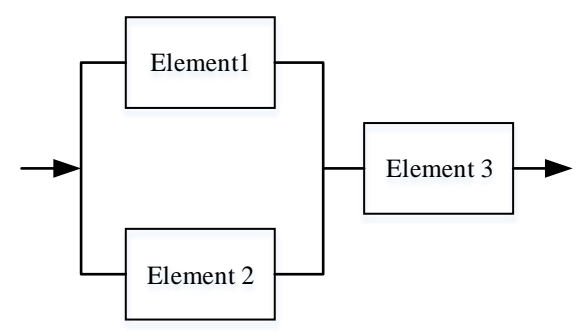

Figure 1. A Flow Transmission System

Table 1. The Parameters for The Elements.

\begin{tabular}{llll}
\hline$j$ & 1 & 2 & 3 \\
\hline$\tilde{s}_{j 1}$ & 1.4 & 2 & 4 \\
$\tilde{s}_{j 2}$ & $(0.9,1,1.1)$ & $(1.1,1.5,1.7)$ & 0 \\
$\tilde{s}_{j 3}$ & 0 & 0 & $\backslash$ \\
$\tilde{p}_{j 1}$ & $(0.69,0.7,0.71)$ & $(0.59,0.7,0.705)$ & $(0.78,0.8,0.81)$ \\
$\tilde{p}_{j 2}$ & $(0.05,0.1,0.2)$ & $(0.18,0.2,0.22)$ & $(0.19,0.2,0.22)$ \\
$\tilde{p}_{j 3}$ & $(0.15,0.2,0.3)$ & $(0.08,0.1,0.11)$ & $\backslash$ \\
\hline
\end{tabular}

The FUGF of each element is defined as

$$
\begin{aligned}
u_{\tilde{s}_{1}}(z)= & \tilde{p}_{11} z^{\tilde{s}_{11}}+\tilde{p}_{12} z^{\tilde{s}_{12}}+\tilde{p}_{13} z^{\tilde{s}_{13}} \\
= & (0.69,0.7,0.71) z^{1.4}+(0.05,0.1,0.2) z^{(0.9,1,1.1)} \\
& +(0.15,0.2,0.3) z^{0} \\
u_{\tilde{s}_{2}}(z)= & \tilde{p}_{21} z^{\tilde{s}_{21}}+\tilde{p}_{22} z^{\tilde{s}_{22}}+\tilde{p}_{23} z^{\tilde{s}_{23}} \\
= & (0.59,0.7,0.705) z^{2}+(0.18,0.2,0.22) z^{(1.1,1.5,1.7)} \\
& +(0.08,0.1,0.11) z^{0} \\
u_{\tilde{s}_{3}}(z)= & \tilde{p}_{31} z^{\tilde{s}_{31}}+\tilde{p}_{32} z^{\tilde{3}_{32}} \\
= & (0.78,0.8,0.81) z^{4}+(0.19,0.2,0.22) z^{0}
\end{aligned}
$$

By defining the composition operator $\otimes_{\tilde{p}}$, The FUGF for element 1 and element 2 connected in parallel can be calculated as

$$
\begin{aligned}
& \otimes_{\tilde{P}}\left(\tilde{S}_{1}, \tilde{S}_{2}\right)=(0.4071,0.49,0.5006) \cdot z^{3.4} \\
& +(0.1242,0.14,0.1562) \cdot z^{(2.5,2.9,3.1)}+(0.0552,0.07,0.0781) \cdot z^{1.4} \\
& +(0.0295,0.07,0.141) \cdot z^{(2.9,3,3.1)}+(0.009,0.02,0.044) \cdot z^{(2,2.5,2.8)} \\
& +(0.004,0.01,0.022) \cdot z^{(0.9,1,1.1)}+(0.0885,0.14,0.2115) \cdot z^{2} \\
& +(0.027,0.04,0.066) \cdot z^{(1.1,1.5,1.7)}+(0.012,0.02,0.033) \cdot z^{0}
\end{aligned}
$$

The operator $\otimes_{\tilde{S}}$ is applied to obtain the FUGF for the entire system, where element 3 connected in series with the other two elements 1 and 2 which connected in parallel.

$$
\begin{aligned}
& \otimes_{\tilde{S}}\left(\otimes_{\tilde{P}}\left(\tilde{S}_{1}, \tilde{S}_{2}\right), \tilde{S}_{3}\right) \\
& =\sum_{i=1}^{9} \tilde{p}_{i} \cdot z^{\tilde{z}_{i}} \\
& =(0.3175,0.392,0.4055) \cdot z^{3.4}+(0.023,0.056,0.1142) \cdot z^{(2.9,3,3.1)} \\
& +(0.0969,0.112,0.1265) \cdot z^{(2.5,2.9,3.1)} \\
& +(0.007,0.016,0.0356) \cdot z^{(2,2.5,2.8)}+(0.069,0.112,0.1713) \cdot z^{2} \\
& +(0.0211,0.032,0.0535) \cdot z^{(1.1,1.5,1.7)}+(0.0431,0.056,0.0633) \cdot z^{1.4} \\
& +(0.0031,0.008,0.0178) \cdot z^{(0.9,1,1.1)}+(0.1531,0.216,0.3017) \cdot z^{0}
\end{aligned}
$$

For states $(i=1,2,3,4,5), \tilde{g}_{i}>\tilde{\omega}$ definitely, $r_{\text {rel }}^{i}=1$, These states are successful states.

For states $(i=8,9), \quad \tilde{g}_{i}<\tilde{\omega}$ definitely, $r_{r e l}^{i}=0$, These states are failure states.

For state $(i=6,7)$, the state of system is uncertain. $\tilde{g}_{6}=(-0.1,0,0.1), \tilde{g}_{7}=(-0.4,0.1,0.4)$. We can calculate $r_{\text {rel }}^{6}=\frac{0.5 \times[0.1-(-0.1)] \times 1-0.5 \times[0-(-0.1)] \times 1}{0.5 \times[0.1-(-0.1)] \times 1}=0.5$ $r_{\text {rel }}^{7}=\frac{0.5 \times[0.4-(-0.4)] \times 1-0.5 \times[0-(-0.4)] \times 0.8}{0.5 \times[0.4-(-0.4)] \times 1}=0.6$

The fuzzy reliability of the system is calculated as 


$$
\begin{aligned}
\tilde{R}= & (0.3175,0.392,0.4055)+(0.023,0.056,0.1142) \\
& +(0.0969,0.112,0.1265)+(0.007,0.016,0.0356) \\
& +(0.069,0.112,0.1713)+(0.0211,0.032,0.0535) \cdot 0.6 \\
& +0+0 \\
= & (0.5261,0.7072,0.8852)
\end{aligned}
$$

Suppose that the system safety standard requires a reliability which is set as 0.9 . The calculation results shows that the system cannot satisfy the safety standard.

\section{Conclusions}

This paper proposes a fuzzy reliability calculation method based on the fuzzy universal generating function. Though defining different operators, the FUGF of the system can be deduced. The fuzzy reliability of the system is calculated after a given required performance. The proposed method can be used for fuzzy reliability assessment and through comparison, we can know whether the design scheme is feasible.

\section{Acknowledgements}

This research was supported by the Sichuan Science and Technology Program, grant nos. 2019YJ0395 and 2021YJ0519 and General Aviation Special Research Project of Civil Aviation Flight University of China, grant no. THZX2018-09.

\section{Conflict of Interest Statement}

The authors declare no conflict of interest.

\section{References}

[1] I. A. Ushakov, A universal generating function, Soviet Journal of Computer and Systems Sciences 24 (1986) 118-129.

[2] A. Lisnianski, G. Levitin, H. Ben-Haim, Structure optimization of multi-state system with time redundancy, Reliability Engineering \& System Safety 67 (2000) 103-112.

[3] G. Levitin, A. Lisnianski, Joint redundancy and maintenance optimization for multi-state series-parallel systems, Reliability Engineering \& System Safety 64 (1999) 33-42.

[4] G. Levitin, A. Lisnianski, H. Ben-Haim, D. Elmakis, Redundancy optimization for series-parallel multi-state systems, IEEE Transactions on Reliability 47 (1998) 165-172.

[5] W. C. Yeh, The k-out-of-n acyclic multistate-node network reliability evaluation using the universal generating function method, Reliability Engineering \& System Safety 91 (2006) 800-808.

[6] W. C. Yeh, A simple universal generating function method for estimating general multistate-node networks reliability, IIE Transactions 41 (2008) 3-11.

[7] G. Levitin, Y. S. Dai, H. Ben-Haim, Reliability and performance of star topology grid service with precedent constraints on subtask execution, IEEE Transactions on Reliability 55 (2006) 507-515.

[8] W. Li, M. J. Zuo, Reliability evaluation of multi-state weighted k-out-of-n systems, Reliability Engineering \& System Safety 93 (2009) 160-167.

[9] G. Levitin, Y. S. Dai, Optimal service task partition and distribution in grid system with star topology, Reliability Engineering \& Systems Safety 93 (2008) 152-159.
[10] G. Levitin, Optimal allocation of multi-state retransmitters in acyclic transmission network, Reliability Engineering \& System Safety 75 (2002) 73-82.

[11] F. J. Golrokh, G. Azeem, A. Hasan, Eco-efficiency evaluation in cement industries: DEA Malmquist productivity index using optimization models, ENG Transactions 1 (2020) 1-8.

[12] W. Kuo, R. Wan, Recent advances in optimal reliability allocation, IEEE Transactions on Systems, Man, and Cybernetics-Part A: Systems and Humans 37 (2007) 143-156. 